\title{
Gastric tube reconstruction prevents postoperative recurrence and metastasis of esophageal cancer
}

\author{
RUNQI ZHANG, PENG WANG, XIAOYAN ZHANG, LEI ZHANG and CHAO LI \\ Department of Thorax Surgery, Taian Central Hospital, Taian, Shandong 271000, P.R. China
}

Received November 23, 2014; Accepted October 9, 2015

DOI: $10.3892 / 01.2016 .4240$

\begin{abstract}
Esophagectomy is the main method of treating patients with esophageal cancer. Tubular stomach and whole stomach approaches may be used for esophagectomy. However, it is not known to what extent these surgical methods are associated with postoperative recurrence and metastasis. Therefore, we aimed to investigate the effect of the tubular and whole stomach approaches on postoperative recurrence and metastasis in esophageal cancer patients. One hundred and twenty-one patients that were diagnosed with esophageal cancer by gastroscopic biopsy between March 2010 and March 2011 in Taian Central Hospital, China, were recruited into this study. There were 67 cases in the gastric tube group and 54 cases in whole stomach group. All of the patients underwent esophagectomy and there were no mortalities during surgery. All patients completed the follow-up period. The rates of recurrence or metastasis 1 and 2 years after surgery in the gastric tube group were observed to be lower than those in the whole stomach group. The two-year survival rates of the gastric tube group and whole stomach group were 80 and $61 \%$, respectively. There was a significant difference in the survival rate between the two groups $(\mathrm{P}=0.016)$. In conclusion, this study suggests that esophageal cancer patients may gain a mid-term benefit from gastric tube reconstruction.
\end{abstract}

\section{Introduction}

Esophageal cancer is the eighth most common type of cancer and the sixth most common cause of cancer-related mortality worldwide (1). To date, surgical resection remains the main method of treatment for esophageal cancer patients, although the long-term survival rate remains low, even for localized diseases (2). Currently, the tubular stomach and whole stomach approaches are used for esophagectomy in patients with esophageal cancer $(3,4)$. It has been reported that the

Correspondence to: Dr Runqi Zhang, Department of Thorax Surgery, Taian Central Hospital, 29 Longtan Road, Taian, Shandong 271000, P.R. China

E-mail: runqi_zhangta@126.com

Key words: esophageal cancer, gastric tube, metastasis, recurrence tubular stomach method is more effective than the whole stomach method for esophageal reconstruction in terms of dealing with postoperative anastomotic leakage, intrathoracic syndrome and reflux esophagitis (4). However, the extent to which this surgical method is correlated with postoperative recurrence and metastasis remains unknown. Therefore, in the present study we aimed to investigate the effect of the tubular and whole stomach methods on postoperative recurrence and metastasis in esophageal cancer patients who underwent esophagectomy.

\section{Patients and methods}

Patients. A total of 121 patients that were diagnosed with middle (70 cases) and lower (51 cases) esophageal cancer by gastroscopic biopsy between March 2010 and March 2011 at Taian Central Hospital (Taian, China), were recruited into this study. The patients were assigned into the gastric tube group (67 cases; male, 43; female, 24; age, 48-78 years; average age, 62 years) and whole stomach group (54 cases; male, 36; female, 18; age, 51-76 years; average age, 63 years) according to the order of admission. None of the patients received chemotherapy or radiotherapy prior to surgery. Further details of the patient characteristics are shown in Table I. Written informed consent was obtained from all patients.

Surgical methods. All patients underwent esophagectomy through the left thorax, above the aortic arches. All surgeries followed the principles of radical resection of the esophagus: stumping from the esophageal tumor edge was $>5 \mathrm{~cm}$, and wild lymph node dissection was performed at the same time. The tubular stomach approach was used for the patients in the gastric tube group. The tubular stomach was formed from the distal aspect of the lesser curvature of the stomach with the application of linear staplers. It was created by resection of the lesser curvature of the stomach. The formation of the gastric conduit (4-6 cm in diameter) was based on the preservation of the gastroepiploic vessels of the greater curvature of the stomach. Then, the tubular stomach was pulled upwards to the left cervical part through the posterior mediastinal route and hand-sewn two-layer anastomosis was performed. The patients in the whole stomach group underwent the full stomach approach, whereby the stomach was pulled to the left cervical part through the posterior mediastinal route, and hand-sewn two-layer anastomosis was performed. 
Table I. Characteristics of esophageal cancer patients enrolled in the study.

\begin{tabular}{|c|c|c|c|c|}
\hline Characteristic & Gastric tube (cases) & Whole stomach (cases) & Total (cases) & P-value \\
\hline \multicolumn{5}{|l|}{ Age (years) } \\
\hline$\leq 50$ & 2 & 0 & 2 & 0.200 \\
\hline $51-60$ & 23 & 24 & 47 & 0.256 \\
\hline $61-70$ & 33 & 20 & 53 & 0.178 \\
\hline$\geq 71$ & 9 & 10 & 19 & 0.445 \\
\hline \multicolumn{5}{|l|}{ Gender } \\
\hline Male & 43 & 36 & 79 & 0.775 \\
\hline Female & 24 & 18 & 42 & \\
\hline \multicolumn{5}{|l|}{ Tumor location } \\
\hline Middle esophagus & 38 & 32 & 70 & 0.778 \\
\hline Lower esophagus & 29 & 22 & 51 & \\
\hline \multicolumn{5}{|l|}{ Stage } \\
\hline 0 & 0 & 1 & 1 & 0.263 \\
\hline I & 3 & 2 & 5 & 0.832 \\
\hline II & 41 & 35 & 76 & 0.682 \\
\hline III & 23 & 16 & 39 & 0.582 \\
\hline
\end{tabular}

Table II. Fistula and postoperative recurrence or metastasis in esophageal cancer patients.

\begin{tabular}{lccc}
\hline Result & $\begin{array}{c}\text { Gastric } \\
\text { tube }\end{array}$ & $\begin{array}{c}\text { Whole } \\
\text { stomach }\end{array}$ & P-value \\
\hline Gastric fistula & 2 & 0 & 0.200 \\
$\begin{array}{l}\text { Anastomotic fistula } \\
\text { Recurrence or metastasis }\end{array}$ & 1 & 3 & 0.214 \\
$\begin{array}{l}\text { Half a year } \\
\text { 1 year } \\
\text { 2 year }\end{array}$ & 1 & 2 & 0.437 \\
\hline
\end{tabular}

Assessment of recurrence and metastasis. Following surgery, the patients were followed up every 6 months for 2 years. Tests included chest and upper abdominal enhancement computed tomography, emission computed tomography and gastroscopy. Lymph nodes were determined to be enlarged if their diameter was $>1 \mathrm{~cm}$.

Statistical analysis. The SPSS 13.0 statistical package (SPSS Inc., Chicago, IL, USA) was used for the statistical analysis. Measurement data were expressed as the means \pm standard deviation and analyzed by Student's t-test. Enumeration data were calculated by the Chi-square test. The survival analysis was performed using the Kaplan-Meier method and the difference was assessed by the log-rank test. $\mathrm{P}<0.05$ was considered to indicate a statistically significant difference.

\section{Results}

There were no mortalities during surgery, and all of the patients recovered and completed the follow-up period. There

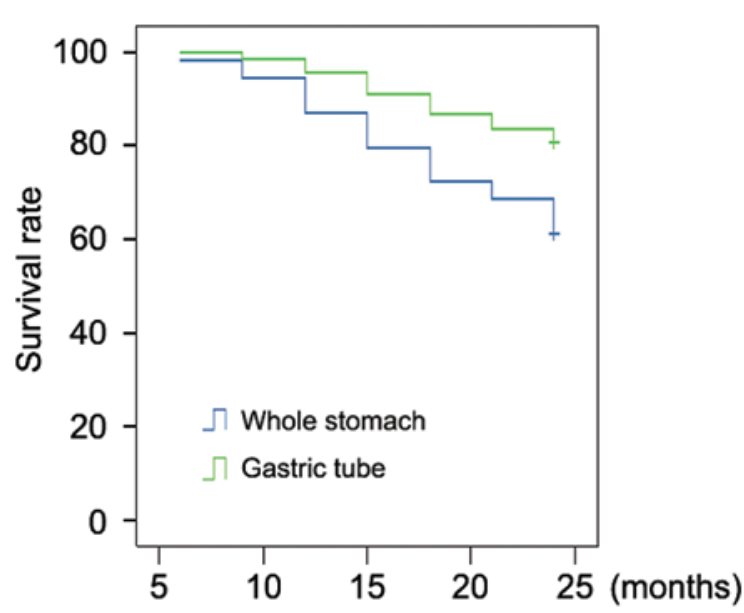

Figure 1. Survival rate of esophageal cancer patients.

was one case of anastomotic fistula and two cases of gastric fistula in the gastric tube group, and three cases of anastomotic fistula in whole stomach group (Table II). All of the patients recovered within 2 months following adequate drainage, irrigation and nutrition support postoperation. With the exception of six patients in stage 0 -I, all patients underwent radiotherapy or chemotherapy according to the pathological results. There was no significant difference between the two groups in terms of the rates of recurrence or metastasis 6 months after surgery (Table II). However, the rates of recurrence or metastasis 1 year and 2 years after surgery in the gastric tube group were lower than those in the whole stomach group (Table II). There were three and seven cases of mortality 1 year after surgery in the gastric tube and whole stomach group, respectively. In the two-year follow-up period, there were 34 cases of mortality due to local tumor recurrence or metastasis; 13 cases in the gastric tube group and 21 cases in the whole stomach group. 
The survival rates of the gastric tube and whole stomach groups were 80 and $61 \%$, respectively (Fig. 1). A significant difference in the survival rate was identified between the two groups $(\mathrm{P}=0.016$; Fig. 1).

\section{Discussion}

In the present study, the data revealed that patients in the gastric tube group had a lower rate of recurrence or metastasis and a higher survival rate compared with the patients in the whole stomach group. Our results suggest that patients with esophageal cancer may obtain mid-term benefits from tubular stomach reconstruction.

The presence of lymph node metastases affects the prognosis of patients with esophageal cancer. It was demonstrated that the lower the location, the lower the grade and the greater the depth of invasion, the more likely a patient was to have independent lymph node metastasis, while there was no significant difference in lymphatic metastasis status among the four stages of esophageal cancer (5). Van de Ven et al (6) reported that lymph node metastasis of thoracic esophageal cancer frequently occurred on the cervicothoracic junction of the recurrent laryngeal nerve chain lymph node, paraesophageal lymph nodes and the cardia $\rightarrow$ left gastric artery $\rightarrow$ celiac lymph node chain. Currently, surgical resection and subsequent lymph node dissection remain the most significant and effective methods in the treatment of esophageal cancer. Abdominal lymph node metastasis of thoracic esophageal cancer normally involves the lesser curvature of the stomach. Recurrent cancer in the thoracic stomach following surgical treatment for esophageal cancer is mainly due to the incomplete resection of lymph nodes in the lesser curvature region. The lesser curvature of the stomach and the cardia are resected in the gastric tube reconstruction surgery, which reduces the recurrence of the gastric tumor. Complete abdominal lymph node dissection may also reduce the risk of postsurgical chest and abdominal lymph node recurrence or metastasis. In addition, the resection of the lesser curvature may help to remove more fat and more of the lymphatic network, and subsequently lead to thorough resection of the omentum lymphatic network and microlymphatic metastasis. Therefore, resection of the lesser curvature may increase the extent of lymph node dissection and decrease the risk of local recurrence and metastasis. The advantages of gastric tube reconstruction, including the ease of surgery, reduction in thoracic stomach syndrome and reflux, and improved quality of life of patients following surgery, have been widely recognized in the clinical treatment of esophageal cancer $(7,8)$. In the present study, our data revealed that the rates of recurrence or metastasis one and two years after surgery in the gastric tube group were lower than those in the whole stomach group. The two-year survival rate of the gastric tube group was also higher than that of the whole stomach group. These data indicate that esophageal cancer patients may obtain greater benefit from the tubular stomach approach compared with the whole stomach approach.

In conclusion, this study demonstrated that the gastric tube may decrease the rate of recurrence or metastasis and improve the two-year survival rate following esophagectomy in esophageal cancer patients. The current study suggests that esophageal cancer patients may gain a mid-term benefit from gastric tube reconstruction.

\section{References}

1. Jemal A, Bray F, Center MM, Ferlay J, Ward E and Forman D: Global cancer statistics. CA Cancer J Clin 61: 69-90, 2011.

2. Gaur P, Kim MP and Dunkin BJ: Esophageal cancer: recent advances in screening, targeted therapy, and management. J Carcinog 13: 11, 2014.

3. Shen C, Yang H, Zhang B, Chen H, Chen Z and Chen J: Improved quality of life in patients with adenocarcinoma of esophagogastric junction after gastric tube reconstruction. Hepatogastroenterology 60: 1985-1989, 2013.

4. Shu YS, Sun C, Shi WP, Shi HC, Lu SC and Wang K: Tubular stomach or whole stomach for esophagectomy through cervico-thoraco-abdominal approach: a comparative clinical study on anastomotic leakage. Ir J Med Sci 182: 477-480, 2013.

5. Ge H, Liu C, Qiu R, Lu Y, Ye K, Yang C, Liu X, Zheng X and Zhai $\mathrm{C}$ : The pattern of lymphatic metastasis and influencing factors of thoracic esophageal squamous cell carcinoma. Pract Radiat Oncol 3 (2 Suppl 1): S32-S33, 2013.

6. van de Ven C, De Leyn P, Coosemans W, Van Raemdonck D and Lerut T: Three-field lymphadenectomy and pattern of lymph node spread in T3 adenocarcinoma of the distal esophagus and the gastro-esophageal junction. Eur J Cardiothorac Surg 15: 769-773, 1999.

7. Okamoto N, Ozawa S, Kitagawa Y, Shimizu Y and Kitajima M: Metachronous gastric carcinoma from a gastric tube after radical surgery for esophageal carcinoma. Ann Thorac Surg 77: 1189-1192, 2004.

8. Ninomiya I, Okamoto K, Oyama K, Hayashi H, Miyashita T, Tajima H, Kitagawa H, Fushida S, Fujimura T and Ohta T: Feasibility of esophageal reconstruction using a pedicled jejunum with intrathoracic esophagojejunostomy in the upper mediastinum for esophageal cancer. Gen Thorac Cardiovasc Surg 62: 627-634, 2014. 